Original Article Journal of Epilepsy Research pISSN 2233-6249 / elSSN 2233-6257

Received April 18, 2018

Revised July 25, 2018

Accepted August 3, 2018

Corresponding author:

Sung-Pa Park, MD, PhD

Department of Neurology, School of Medicine,

Kyungpook National University, 680

Gukchaebosang-ro, Jung-gu, Daegu 41944,

Korea

Tel. $+82-53-420-5769$

Fax. +82-53-422-4265

E-mail; sppark@mail.knu.ac.kr

\section{Validity of the Liverpool Adverse Events Profile as a Screening Tool for Detecting Comorbid Depression or Anxiety Disorder in People with Epilepsy}

\author{
Oh-Young Kwon, MD, PhD' , Sung-Pa Park, MD, PhD² \\ ${ }^{1}$ Department of Neurology and Institute of Health Science, Gyeongsang National University Hospital, Gyeongsang \\ National University College of Medicine, Jinju; ${ }^{2}$ Department of Neurology, School of Medicine, Kyungpook National \\ University, Daegu, Korea
}

\begin{abstract}
Background and Purpose: The Liverpool adverse events profile (LAEP) is useful for detecting and monitoring the adverse effects of antiepileptic drugs (AEDs) and contains items related to symptoms of depression or anxiety. This study evaluated the usefulness of the LAEP for detecting comorbid depression or anxiety disorder in people with epilepsy (PWE).
\end{abstract}

Methods: PWE, aged from 18 to 70 years and who took AEDs for at least 1 year, were included. They completed the Korean version of the LAEP (K-LAEP) to detect the adverse effects of AEDs and the mini international neuropsychiatric interview-plus version 5.0.0 (MINI) to identify major depressive disorder (MDD) and generalized anxiety disorder (GAD). We validated the K-LAEP as a screening tool for coexisting MDD or GAD in PWE. Receiver operating character (ROC) curve analyses were used to measure the appropriateness of cutoff scores for the total and item K-LAEP scores for detecting MDD or GAD.

Results: The study enrolled 150 PWE. According to the MINI, 30 PWE (20.0\%) had MDD and 26 (17.3\%) had GAD. Cronbach's $\alpha$ coefficient of the K-LAEP was 0.939 . For the total K-LAEP score, cutoff scores of 40 and 43 could detect MDD and GAD, respectively. For the K-LAEP item score, a cutoff of five could detect MDD or GAD.

Conclusions: The K-LAEP is a valid screening tool for detecting MDD and GAD in PWE. A high LAEP score suggests comorbid psychiatric disorders, which need further specific evaluation. (2018;8:74-80)

Key words: Epilepsy, Depression, Anxiety, Anticonvulsants, Drug-related side effects and adverse reactions, Psychiatric status rating scales

\section{Introduction}

The goal of the management of people with epilepsy (PWE) is to control seizures and improve their quality of life (QOL). However, adverse effects of antiepileptic drugs (AEDs) can significantly impair QOL. ${ }^{1}$ Hence, it is very important to identify and reduce the adverse effects of AEDs in epilepsy treatment. ${ }^{2}$ The Liverpool adverse events profile (LAEP) is a 19-item self-report questionnaire that is used to screen adverse effects of AEDs. ${ }^{3}$ The validity and reliability of various versions of the LAEP have been verified, including English, Spanish, and Korean language versions. ${ }^{2,45}$

The LAEP is useful for detecting and monitoring the adverse effects of AEDs and there is considerable evidence that the total LAEP score is high in depressed or anxious PWE. ${ }^{4-9}$ This might be because the LAEP contains items pertaining to emotional and psychosomatic symptoms related to depression or anxiety. Conversely, the LAEP may be useful for screening major depression and anxiety.

Psychiatric comorbidities of PWE have negative impacts on outcomes and QOL. To simultaneously identify the psychiatric comorbidities and adverse effects of AEDs, clinicians can administer simple questionnaires, such as the neurological disorders depression inventory for epilepsy (NDDI-E) ${ }^{10}$ and the generalized anxiety disorder-7 (GAD-7), ${ }^{11}$ together with the LAEP. However, administering all three inventories at the same time may unnecessarily burden busy epilepsy clinics, although they are rapid screening tools. If the diagnostic value of the LAEP for major depressive disorder (MDD) or gen- 
eralized anxiety disorder (GAD) is similar to that of the NDDI-E or GAD-7, then we can use the LAEP exclusively for screening MDD or $G A D$, as well as the adverse effects of AEDs. For this reason, this study evaluated the usefulness of the LAEP for screening MDD and GAD.

\section{Methods}

\section{Subjects}

Consecutive PWE who had been treated with AEDs for at least 1 year at the epilepsy clinic at Kyungpook National University Hospital since October 2016 were invited to join the study. Patients who were 18 to 70 years old and had a current diagnosis of epilepsy were included. Diagnosis of epilepsy and classification of epilepsy syndrome were performed according to the criteria of the international league against epilepsy (ILAE). ${ }^{12}$

Patients with an intellectual disability or serious medical, neurological, or psychiatric disorder, or other disorders that prevented them from understanding the questionnaire and cooperating with the interviewer, were excluded. PWE who did not have serious disorders and could cooperate with the interview were not excluded even when taking antidepressants. People with post-stroke epilepsy or history of stroke were not excluded if the stroke status was not serious and they could cooperate the interview.

\section{Study design}

The Institutional Review Board (IRB) of Kyungpook National University Hospital approved this cross-sectional study. The IRB number was KNUH 2015-03018. Informed consent was obtained from all participants at the time of enrollment. Demographic and clinical information were collected at an interview in the clinic, and all data were entered into a computer file.

Epilepsy syndrome is of four types: temporal lobe epilepsy (TLE), extra-TLE (epilepsy syndromes in which the epileptic attacks originate from the frontal, parietal, or occipital lobes), generalized epilepsy, and unknown syndrome. The AED regimen was classified as monotherapy or polytherapy according to the number of AEDs the patient was using. Patients were classified into three categories based on seizure control status: uncontrolled (UCE), well-controlled (WCE), and poorly controlled (PCE) epilepsy. UCE (i.e., drug-refractory epilepsy) was defined as an average of more than one seizure per month for 18 months and a maximum seizure-free period of less than 3 months. WCE was defined as freedom from seizures dur- ing the preceding year, and PCE was defined as an intermediate degree of seizure control that did not meet the criteria for UCE or WCE. ${ }^{13}$ The seizure control category of each epilepsy patient was determined based on the seizure frequency documented in their medical records.

Eligible patients completed the Korean version of the LAEP $(K-L A E P)^{5}$ and the mini international neuropsychiatric interview-plus version 5.0.0 (MINI). ${ }^{14}$ To measure the validity of the K-LAEP for diagnosing MDD and GAD, a neuropsychologist examined their current $M D D$, and GAD using the MINI.

\section{Questionnaire and interview}

\section{K-LAEP}

The K-LAEP is a 19-item self-report questionnaire used to identify and monitor the frequency and severity of common adverse effects associated with AEDs in Korean PWE. ${ }^{4}$ This questionnaire asks PWE how often adverse effects of AEDs occurred during the past 4 weeks. Each item is scored on a four-point Likert scale: 1, never a problem; 2, rarely a problem; 3 , sometimes a problem; and 4 , often or always a problem. The total score ranges from 19 to 76 , and higher scores are indicative of a greater burden from the adverse effects. The Cronbach's $\alpha$ coefficient of the K-LAEP is 0.9 . We used the 21-item version, which includes two additional items (thinking unclearly and slurred speech) for the QOL study, ${ }^{1}$ the total possible K-LAEP score in our study ranged from 21 to 84 . The item score was defined as the number of items that were scored three or four points.

\section{MINI}

The MINI allows a brief and structured clinical interview, and represents the gold standard test for detecting psychiatric disorders based on the Diagnosis and Statistical Manual of Mental Disorders-IV criteria. ${ }^{15}$ The Korean version of the MINI has been validated. ${ }^{14}$ The Cohen's kappa values for diagnosing MDD and GAD using the Korean version of the MINI were 0.71 and 0.57 , respectively. Among the various modules of MINI, we used the current time frame of major depressive episode module for MDD diagnosis and the GAD module for GAD diagnosis.

\section{Statistical analysis}

The SPSS software package (version 23.0; SPSS Inc., Chicago, IL, USA) was used for the data analysis. MedCalc (version 8.0; MedCalc Software, Mariakerke, Belgium) was used to perform receiver operating characteristic (ROC) analyses, which can provide measures of 
sensitivity, specificity, positive predictive value (PPV) and negative predictive value (NPV). ROC analyses of the total and item scores of the K-LAEP, over a range of cutoff scores, were performed to compare the MDD and GAD diagnoses with those obtained with the MINI. Optimal cutoff scores were also computed using criteria that minimize the Euclidean distance from point (sensitivity and specificity) to point in the $x$-y plane. Cronbach's a was computed as a measure of internal consistency and was recalculated after items were removed. The significance level of the statistical measures was set at $p<0.05$.

Table 1. Demographic and clinical characteristics of eligible patients $(n=150)$

\begin{tabular}{|c|c|}
\hline Characteristic & Value \\
\hline Age (years) & $41.6 \pm 12.2(18-70)$ \\
\hline Gender (male) & $94(62.7)$ \\
\hline Education (years) & $12.9 \pm 2.8(6-18)$ \\
\hline Age at onset (years) & $23.6 \pm 13.4(1-67)$ \\
\hline Duration of epilepsy (years) & $18.0 \pm 10.4(1-50)$ \\
\hline Type of seizure (partial) & $117(78.0)$ \\
\hline \multicolumn{2}{|l|}{ Epilepsy syndrome } \\
\hline Temporal lobe epilepsy & $74(49.3)$ \\
\hline Extra-temporal lobe epilepsy & $43(28.7)$ \\
\hline Generalized epilepsy & $28(18.7)$ \\
\hline Unknown & $5(3.3)$ \\
\hline \multicolumn{2}{|l|}{ Seizure control } \\
\hline Well-controlled epilepsy & $87(58.0)$ \\
\hline Poorly controlled epilepsy & $40(26.7)$ \\
\hline Uncontrolled epilepsy & $23(15.3)$ \\
\hline MRI, abnormal & $73(48.7)$ \\
\hline History of febrile convulsion & $38(25.3)$ \\
\hline Family history of epilepsy & $6(4.0)$ \\
\hline Duration of AED intake (years) & $15.0 \pm 10.0(1-50)$ \\
\hline AED regimen, monotherapy & $70(46.7)$ \\
\hline MDD by $\mathrm{MINI}$ & $30(20.0)$ \\
\hline GAD by MINI & $26(17.3)$ \\
\hline Total score of K-LAEP & $36.7 \pm 13.2(21-76)$ \\
\hline Number of items in K-LAEP* & $4.5 \pm 5.0(0-20)$ \\
\hline \multicolumn{2}{|c|}{$\begin{array}{l}\text { Values are presented as mean } \pm \text { standard deviation (range) or number (\%). } \\
\text { MRI, magnetic resonance imaging; AED, antiepileptic drug; MDD, major } \\
\text { depressive disorder; MINI, mini international neuropsychiatric interview-plus } \\
\text { version 5.0.0; GAD, generalized anxiety disorder; K-LAEP, Korean version } \\
\text { of Liverpool adverse event profile. } \\
\text { *The number of items that was given three or four points among } 21 \\
\text { items of the K-LAEP. }\end{array}$} \\
\hline
\end{tabular}

\section{Results}

Initially, 182 patients visited the clinic, but 32 were excluded because they refused to participate $(n=12)$, had a shorter duration of AED use $(n=6)$, had an intellectual disability $(n=6)$, or were too young $(n=5)$ or too old $(n=3)$. After all, 150 patients participated in this study.

\section{Frequencies of MDD and GAD determined by the MINI}

Table 1 shows the frequencies of MDD and GAD determined by the MINI. MDD was diagnosed in 30 PWE (20\%), and GAD in 26 $(17.3 \%)$.

\section{Reliability of K-LAEP}

Cronbach's a coefficient of the K-LAEP was 0.939. Corrected item-total correlations of items of the K-LAEP are presented in Table 2.

Table 2. Corrected item-total correlations* and Cronbach's a if an item is deleted from the K-LAEP

\begin{tabular}{lcc}
\hline & $\begin{array}{c}\text { Corrected } \\
\text { item-total } \\
\text { correlation }\end{array}$ & $\begin{array}{c}\text { Cronbach's } \\
\text { alpha if item } \\
\text { deleted }\end{array}$ \\
\hline Unsteadiness & 0.572 & 0.937 \\
Tiredness & 0.678 & 0.936 \\
Restlessness & 0.716 & 0.935 \\
Feeling of anger or aggression to others & 0.725 & 0.935 \\
Nervousness and/or agitation & 0.756 & 0.934 \\
Headache & 0.594 & 0.937 \\
Hair loss & 0.521 & 0.938 \\
Problem with skin (e.g., acne, rash) & 0.465 & 0.939 \\
Double of blurred vision & 0.692 & 0.935 \\
Upset stomach & 0.574 & 0.937 \\
Difficulty in concentrating & 0.722 & 0.935 \\
Trouble with mouth or gums & 0.548 & 0.938 \\
Shaky hands & 0.398 & 0.940 \\
Weight gain & 0.482 & 0.939 \\
Dizziness & 0.675 & 0.936 \\
Sleepiness & 0.641 & 0.936 \\
Depression & 0.723 & 0.935 \\
Memory problem & 0.707 & 0.935 \\
Disturbed sleep & 0.628 & 0.936 \\
Difficulty in thinking & 0.725 & 0.935 \\
Slurred speech & 0.692 & 0.935 \\
\hline K-LAEP, Korean version & & \\
\hline
\end{tabular}

K-LAEP, Korean version of the Liverpool adverse events profile. *Corrected item-total correlation: correlation of the designated item with the sum of the other 20 . 
The corrected item-total correlation is the correlation of the designated item of K-LAEP with the sum of the other 20. When the correlation is low for a item, the Cronbach's value will increase if the item is deleted from the inventory. In this study, Cronbach's $\alpha$ value did not change even if the 21 items of K-LAEP were excluded one by one. Therefore, we could say that all items of the K-LAEP were corre- lated and well-coordinated (Table 2).

\section{Total score of K-LAEP for detecting MDD and GAD}

\section{ROC curve for detecting MDD}

Table 3 shows the results of ROC analysis of the total score of the

Table 3. ROC analyses of the total score of the K-LAEP for detecting MDD as determined by the MINI

\begin{tabular}{lcccccccc}
\hline Cutoff score & Sensitivity & Specificity & PPV & NPV & AUC & SE & $95 \% \mathrm{Cl}$ & $p$-value \\
\hline$>38$ & 83.3 & 75.0 & 45.5 & 94.7 & 0.792 & 0.040 & $0.718-0.854$ & \\
$>39$ & 80.0 & 75.8 & 45.3 & 93.8 & 0.779 & 0.042 & $0.704-0.843$ & \\
$>40$ & 80.0 & 80.0 & 50.0 & 94.1 & 0.800 & 0.041 & $0.727-0.861$ & $<0.001$ \\
$>41$ & 76.7 & 81.7 & 51.1 & 93.3 & 0.792 & 0.043 & $0.718-0.854$ & \\
$>42$ & 76.7 & 83.3 & 53.5 & 93.5 & 0.800 & 0.043 & $0.727-0.861$ & \\
\hline
\end{tabular}

ROC, receiver operating characteristic; K-LAEP, Korean version of the Liverpool adverse events Profile; MDD, major depressive disorder; MINI, mini international neuropsychiatric interview-plus version 5.0.0; PPV, positive predictive value; NPV, negative predictive value; AUC, area under the curve; SE, standard error; $\mathrm{Cl}$, confidence interval.
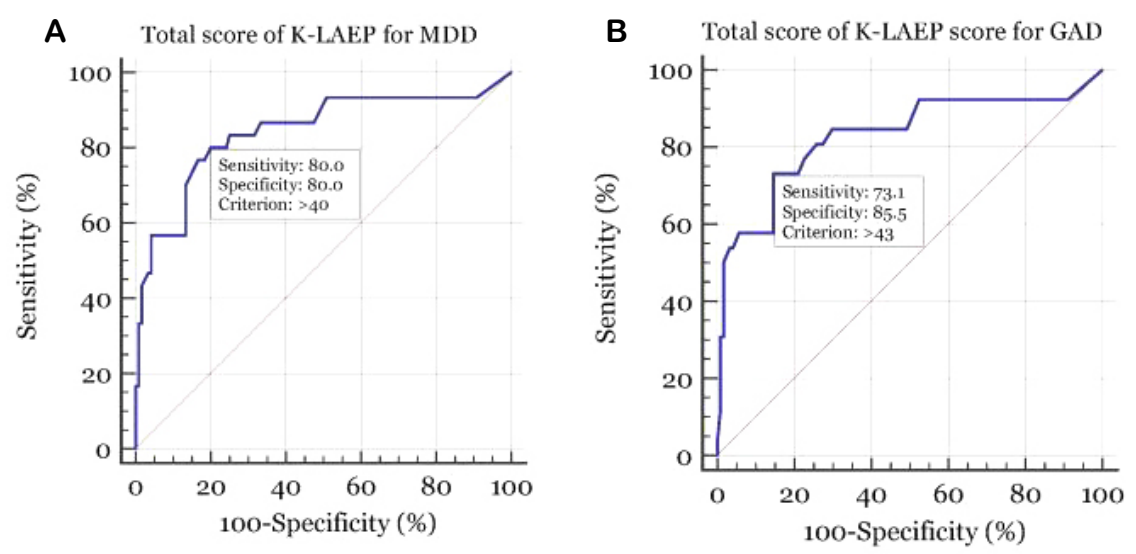

Figure 1. Receiver operating character (ROC) curve of the total score of the Korean version of the Liverpool Adverse Events Profile (K-LAEP) for detecting major depressive disorder (MDD) and generalized anxiety disorder (GAD). For detecting MDD, ROC analysis of the total K-LAEP score yielded an area under the curve (AUC) of 0.800 . At a cutoff score of $>40$, sensitivity and specificity for detecting MDD were $80.0 \%$ together (A). For detecting GAD, ROC analysis of the total score of the K-LAEP determined an AUC of 0.793. At a cutoff score of $>43$, sensitivity and specificity for detecting GAD were $73.1 \%$ and $85.5 \%$ (B).

Table 4. ROC analyses of the total score of the K-LAEP for detecting GAD as determined by the MINI

\begin{tabular}{lcccccccc}
\hline Cutoff score & Sensitivity & Specificity & PPV & NPV & AUC & SE & $95 \% \mathrm{Cl}$ & $p$-value \\
\hline$>41$ & 73.1 & 79.0 & 42.2 & 93.3 & 0.761 & 0.048 & $0.684-0.826$ & \\
$>42$ & 73.1 & 80.7 & 44.2 & 93.5 & 0.769 & 0.048 & $0.693-0.833$ & \\
$>43$ & 73.1 & 85.5 & 51.4 & 93.8 & 0.793 & 0.047 & $0.719-0.855$ & $<0.001$ \\
$>44$ & 57.7 & 85.5 & 45.5 & 90.6 & 0.716 & 0.052 & $0.637-0.786$ & \\
$>45$ & 57.7 & 86.3 & 46.9 & 90.7 & 0.720 & 0.052 & $0.641-0.790$ & \\
\hline
\end{tabular}

ROC, receiver operating characteristic; K-LAEP, Korean version of the Liverpool adverse events profile; GAD, generalized anxiety disorder; MINI, mini international neuropsychiatric interview-plus version 5.0.0; PPV, positive predictive value; NPV, negative predictive value; AUC, area under the curve; $\mathrm{SE}$, standard error; $\mathrm{Cl}$, confidence interval. 
K-LAEP for detecting MDD with the MINI (Fig. 1A). ROC analysis of the total K-LAEP score yielded an area under the curve (AUC) of $0.800(95 \%$ confidence interval $[\mathrm{Cl}]=0.727-0.861$; standard error $[\mathrm{SE}]=0.041 ; p<0.001)$. Using a cutoff score of $>40$, the sensitivity and specificity were both $80.0 \%$, with a PPV of $50.0 \%$ and NPV of $94.1 \%$.

\section{ROC curve for detecting GAD}

Table 4 shows the results of ROC analysis of the total score of the K-LAEP for detecting GAD with the MINI (Fig. 1B). ROC analysis of the total K-LAEP score yielded an AUC of $0.793(95 \% \mathrm{Cl}=$ $0.719-0.855 ; \mathrm{SE}=0.047 ; p<0.001)$. With a cutoff score of $>43$, the sensitivity was $73.1 \%$ and the specificity was $85.5 \%$, with a PPV of $51.4 \%$ and NPV of $93.8 \%$.

Table 5. ROC analyses of the item score of the K-LAEP for detecting MDD as determined by the MINI

\begin{tabular}{lcccccccc}
\hline Cutoff score & Sensitivity & Specificity & PPV & NPV & AUC & SE & $95 \%$ Cl & $p$-value \\
\hline$>3$ & 90.0 & 62.5 & 37.5 & 96.2 & 0.762 & 0.036 & $0.686-0.828$ & \\
$>4$ & 83.3 & 74.2 & 44.6 & 94.7 & 0.788 & 0.040 & $0.713-0.850$ & $0.732-0.864$ \\
$>5$ & 83.3 & 79.6 & 48.1 & 94.9 & 0.804 & 0.040 & 0.001 \\
$>6$ & 73.3 & 85.0 & 55.0 & 92.7 & 0.792 & 0.044 & $0.718-0.854$ \\
$>7$ & 63.3 & 89.2 & 59.4 & 90.7 & 0.762 & 0.047 & $0.686-0.828$ & \\
\hline
\end{tabular}

ROC, receiver operating characteristic; K-LAEP, Korean version of the Liverpool adverse events profile; MDD, major depressive disorder; MINI, mini international neuropsychiatric interview-plus version 5.0.0; PPV, positive predictive value; NPV, negative predictive value; AUC, area under the curve; SE, standard error; $\mathrm{Cl}$, confidence interval.
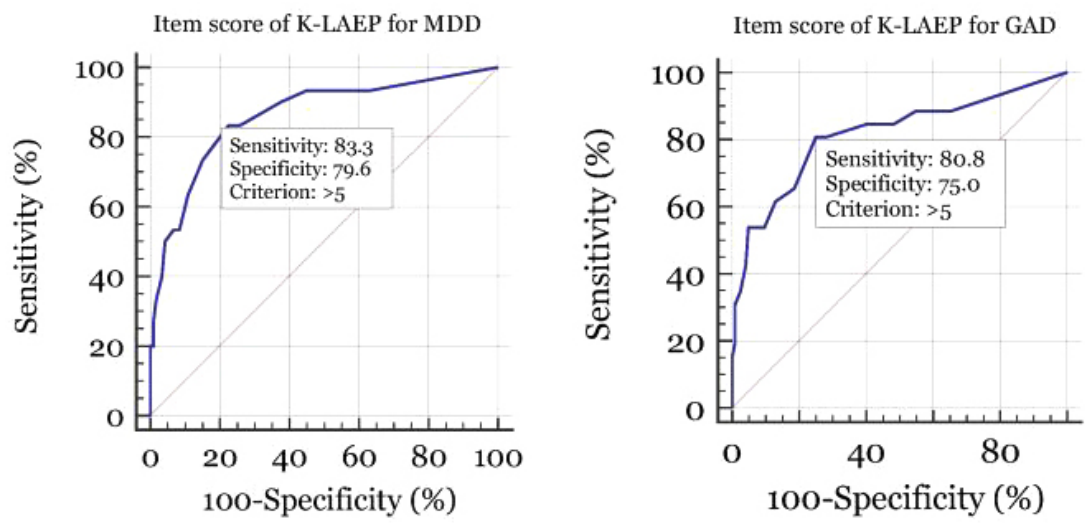

Figure 2. Receiver operating character (ROC) curve of the item score of the Korean version of the Liverpool Adverse Events Profile (K-LAEP) for detecting major depressive disorder (MDD) or generalized anxiety disorder (GAD). For detecting MDD, ROC analysis of the item score of the K-LAEP determined an area under the curve (AUC) of 0.804 . At a cutoff score of $>5$, sensitivity and specificity for detecting MDD were 83.3\% and 79.6\% (A). For detecting GAD, ROC analysis of the item score of the K-LAEP determined an AUC of 0.779 . At a cutoff score of $>5$, sensitivity and specificity for detecting GAD were $80.8 \%$ and $75.0 \%$ (B).

Table 6. ROC analyses of the item score of the K-LAEP for detecting GAD as determined by the MINI

\begin{tabular}{lcccccccc}
\hline Cutoff score & Sensitivity & Specificity & PPV & NPV & AUC & SE & $95 \%$ CI & $p$-value \\
\hline$>3$ & 84.6 & 59.7 & 30.6 & 94.9 & 0.721 & 0.042 & $0.642-0.791$ & \\
$>4$ & 80.8 & 71.8 & 37.5 & 94.7 & 0.763 & 0.044 & $0.686-0.828$ & \\
$>5$ & 80.8 & 75.0 & 40.4 & 94.9 & 0.779 & 0.044 & $0.704-0.842$ & $<0.001$ \\
$>6$ & 65.4 & 81.5 & 42.5 & 91.8 & 0.734 & 0.051 & $0.656-0.803$ & \\
$>7$ & 61.5 & 87.1 & 50.0 & 91.5 & 0.743 & 0.051 & $0.666-0.811$ & \\
\hline
\end{tabular}

ROC, receiver operating characteristic; K-LAEP, Korean version of the Liverpool adverse events profile; GAD, generalized anxiety disorder; MINI, mini international neuropsychiatric interview-plus version 5.0.0; PPV, positive predictive value; NPV, negative predictive value; AUC, area under the curve; $\mathrm{SE}$, standard error; $\mathrm{Cl}$, confidence interval. 


\section{Item score of K-LAEP for detecting for MDD and GAD}

\section{ROC curve for detecting MDD}

Table 5 shows the results of ROC analysis of the item score of the K-LAEP for detecting MDD with the MINI in (Fig. 2A). As we mentioned above, the item score was defined as the number of items of K-LAEP that were scored three or four points. ROC analysis of the K-LAEP item score yielded an AUC of $0.804(95 \% \mathrm{Cl}=0.732-0.864$; $\mathrm{SE}=0.040 ; p<0.001)$. With a cutoff score of $>5$, the sensitivity was $83.3 \%$ and the specificity $79.6 \%$, with a PPV of $48.1 \%$ and NPV of $94.9 \%$.

\section{ROC curve for detecting GAD}

Table 6 shows the results of ROC analysis of the item score of the K-LAEP for detecting GAD with the MINI (Fig. 2B). ROC analysis of the K-LAEP item score yielded an AUC of $0.779(95 \% \mathrm{Cl}=0.704$ $0.842 ; \mathrm{SE}=0.044 ; p<0.001)$. At a cutoff score of $>5$, the sensitivity was $80.8 \%$ and the specificity $75.0 \%$, with a PPV of $40.4 \%$ and NPV of $94.9 \%$.

\section{Discussion}

The treatment goal in epilepsy is to improve QOL as well as to control seizures. However, it is impossible to avoid adverse effects entirely when using AEDs. PWE may also complain of negative events that are not directly related to AEDs, but instead occur coincidentally while using AEDs. ${ }^{9}$ Detection and reduction of these adverse effects is very important in the management of PWE, because they can interfere with the maintenance of effective AEDs and may even reduce QOL. ${ }^{2}$ Reports have shown that depression and anxiety are associated with a high total LAEP score. ${ }^{4-9}$ This being so, clinicians should clarify whether a high total LAEP score is likely to be associated with psychiatric disorders such as MDD and GAD, because these illnesses have a serious impact on QOL and the mortality rate of PWE., ${ }^{816}$

The association between a high total LAEP score and depression and anxiety may be a drawback when using the instrument as a screening tool for common adverse effects of AEDs. Depression and anxiety affect the total LAEP score because it contains items that are related to emotional and psychosomatic symptoms, and depression and anxiety may cause those emotional and psychosomatic symptoms independent of AEDs. Anxiety was even shown to be associated with high scores on items pertaining to acne, hair loss, weight gain, and mouth problems, which are not emotional in nature and do not seem to be associated with psychosomatic symptoms. ${ }^{7}$ On this basis, we hypothesized that the LAEP may be useful for screening for MDD and GAD.

Most outpatient epilepsy clinics are too busy to evaluate all aspects of PWE. If we have a quick inventory that can examine many aspects of PWE, we can efficiently use the inventory in a busy epilepsy clinic. Hypothesizing that the LAEP may be useful for screening for MDD and GAD, we conducted this validation study based on a gold standard test, i.e., the MINI. We found that LAEP was useful for screening for MDD and GAD. Therefore, the LAEP serves not only as a standardized tool for monitoring the common adverse effects of AEDs, but can also be used to screen for MDD and GAD.

Rapid screening of depression and anxiety is more important in UCE than in WCE. Depression and anxiety occur more frequently in PWE whose seizures are not well-controlled. ${ }^{17}$ Patients with newly diagnosed epilepsy are more likely to develop resistance to AEDs when depression and anxiety are present. ${ }^{18,19}$ Depression and anxiety in PWE have also been shown to negatively affect the outcomes of pharmacologic and surgical treatments of epilepsy. ${ }^{20,21}$ For this reason, LAEP would be useful for screening for depression and anxiety in UCE.

We validated that the LAEP is useful for screening MDD and GAD through this study. This study has a fundamental limitation because the LAEP is a inventory developed to monitor common adverse effects of AEDs. In patients without MDD or GAD, multiple side effects from AEDs can be mis-screened for MDD or GAD. Nevertheless, the results of this study suggested that the LAEP could be used as a multimodal screening tool in busy epilepsy clinics.

The LAEP is a standard screening tool for identifying and monitoring common adverse effects of AEDs. It is easy to use the LAEP in a busy epileptic clinic because the LAEP is simple and takes a short time. The LAEP usually takes less than 5 minutes to complete. ${ }^{22}$ Our results, and the rapidity of the test, underscore the usefulness of the LAEP. If the total LAEP score is high in PWE, we should consider comorbid depression and anxiety as well as the adverse effects of AEDs. Taken together, a high LAEP score signifies that depression and anxiety should be investigated further; such investigations could include gold standard tests, such as the MINI, and psychiatric consultation.

\section{Conflicts of Interest}

The authors have no financial or other potential conflicts of interest to declare in relation to this study. 


\section{References}

1. Baker GA, Jacoby A, Buck D, Stalgis C, Monnet D. Quality of life of people with epilepsy: a European study. Epilepsia 1997;38:353-62.

2. Gilliam FG, Fessler AJ, Baker G, Vahle V, Carter J, Attarian H. Systematic screening allows reduction of adverse antiepileptic drug effects: a randomized trial. Neurology 2004;62:23-7.

3. Perucca $P$, Gilliam FG. Adverse effects of antiepileptic drugs. Lancet Neurol 2012;11:792-802.

4. Carreño $M$, Donaire A, Falip M, et al. Validation of the Spanish version of the Liverpool adverse events profile in patients with epilepsy. Epilepsy Behav 2009;15:154-9.

5. Park JM, Seo JG, Park SP. Validity and reliability of the Korean version of the Liverpool adverse events profile (K-LAEP) in people with epilepsy. J Korean Epilep Soc 2012;16:43-8.

6. Kanner AM, Barry JJ, Gilliam F, Hermann B, Meador KJ. Depressive and anxiety disorders in epilepsy: do they differ in their potential to worsen common antiepileptic drug-related adverse events? Epilepsia 2012;53: 1104-8.

7. Kim SK, Park SP, Kwon OY. Impact of depression and anxiety on adverse event profiles in Korean people with epilepsy. Epilepsy Behav 2015;46: 185-91.

8. Lee SJ, Kim JE, Seo JG, et al. Predictors of quality of life and their interrelations in Korean people with epilepsy: a MEPSY study. Seizure 2014; 23:762-8.

9. Perucca P, Jacoby A, Marson AG, et al. Adverse antiepileptic drug effects in new-onset seizures: a case-control study. Neurology 2011;76:273-9.

10. Ko PW, Hwang J, Lim HW, Park SP. Reliability and validity of the Korean version of the Neurological Disorders Depression Inventory for Epilepsy (K-NDDI-E). Epilepsy Behav 2012;25:539-42.

11. Seo JG, Cho YW, Lee SJ, et al. Validation of the generalized anxiety disorder-7 in people with epilepsy: a MEPSY study. Epilepsy Behav 2014;
35:59-63.

12. Proposal for classification of epilepsies and epileptic syndromes. Commission on classification and terminology of the international league against epilepsy. Epilepsia 1985;26:268-78.

13. Berg AT, Shinnar S, Levy SR, Testa FM, Smith-Rapaport S, Beckerman B. Early development of intractable epilepsy in children: a prospective study. Neurology 2001;56:1445-52.

14. Yoo SW, Kim YS, Noh JS, et al. Validity of Korean version of the mini-international neuropsychiatric interview. Anxiety Mood 2006;2:50-5.

15. Jones JE, Hermann BP, Barry JJ, Gilliam FG, Kanner AM, Meador KJ. Rates and risk factors for suicide, suicidal ideation, and suicide attempts in chronic epilepsy. Epilepsy Behav 2003;4 Suppl 3:S31-8.

16. Seo JG, Lee JJ, Cho YW, et al. Suicidality and its risk factors in Korean people with epilepsy: a MEPSY Study. J Clin Neurol 2015;11:32-41.

17. Kwon OY, Park SP. Frequency of affective symptoms and their psychosocial impact in Korean people with epilepsy: a survey at two tertiary care hospitals. Epilepsy Behav 2013;26:51-6.

18. Hitiris N, Mohanraj R, Norrie J, Sills GJ, Brodie MJ. Predictors of pharmacoresistant epilepsy. Epilepsy Res 2007;75:192-6.

19. Petrovski S, Szoeke CE, Jones NC, et al. Neuropsychiatric symptomatology predicts seizure recurrence in newly treated patients. Neurology 2010; 75:1015-21.

20. Cleary RA, Thompson PJ, Fox Z, Foong J. Predictors of psychiatric and seizure outcome following temporal lobe epilepsy surgery. Epilepsia 2012:53:1705-12.

21. de Araújo Filho GM, Gomes FL, Mazetto L, et al. Major depressive disorder as a predictor of a worse seizure outcome one year after surgery in patients with temporal lobe epilepsy and mesial temporal sclerosis. Seizure 2012;21:619-23.

22. Baker GA, Frances $P$, Middleton $E$, et al. Initial development, reliability, and validity of a patient-based adverse drug event scale. Epilepsia 1994;35(Suppl 7):80. 